\title{
BMJ Open COVID-19 international border surveillance at Toronto's Pearson Airport: a cohort study
}

\author{
Vivek Goel (1) , ${ }^{1}$ David Bulir, ${ }^{2}$ Eric De Prophetis, ${ }^{1}$ Munaza Jamil, ${ }^{3}$ \\ Laura C Rosella (D) , ${ }^{1}$ Dominik Mertz (D) , ${ }^{4}$ Cheryl Regehr (D) , ${ }^{5}$ Marek Smieja ${ }^{2}$
}

To cite: Goel V, Bulir D, De Prophetis E, et al. COVID-19 international border surveillance at Toronto's Pearson Airport: a cohort study. BMJ Open 2021;11:e050714. doi:10.1136/ bmjopen-2021-050714

- Prepublication history for this paper is available online To view these files, please visit the journal online (http://dx.doi org/10.1136/bmjopen-2021050714).

Received 26 February 2021 Accepted 14 June 2021

Check for updates

(C) Author(s) (or their employer(s)) 2021. Re-use permitted under CC BY-NC. No commercial re-use. See rights and permissions. Published by BMJ.

${ }^{1}$ Dalla Lana School of Public Health, University of Toronto, Toronto, Ontario, Canada ${ }^{2}$ Department of Pathology and Molecular Medicine, McMaster University Faculty of Health Sciences, Hamilton, Ontario, Canada

${ }^{3}$ McMaster University Faculty of Health Sciences, Hamilton, Ontario, Canada

${ }^{4}$ Division of Infectious Diseases, Department of Medicine,

McMaster University Faculty of Health Sciences, Hamilton, Ontario, Canada

${ }^{5}$ Factor Inwentash Faculty of Social Work, University of Toronto, Toronto, Ontario, Canada

Correspondence to

Dr Vivek Goel;

vivek.goel@utoronto.ca

\section{ABSTRACT}

Objectives The primary objective was to estimate the positivity rate of air travellers coming to Toronto, Canada in September and October 2020, on arrival and on day 7 and day 14 . The secondary objectives were to estimate the degree of risk based on country of origin and to assess knowledge and attitudes towards COVID-19 control measures and subjective well-being during the quarantine period.

Design Prospective cohort of arriving international travellers.

Setting Toronto Pearson Airport Terminal 1, Toronto, Canada.

Participants Participants of this study were passengers arriving on international flights. Inclusion criteria were those aged 18 or older who had a final destination within $100 \mathrm{~km}$ of the airport, spoke English or French, and provided consent. Excluded were those taking a connecting flight, had no internet access, exhibited symptoms of COVID-19 on arrival or were exempted from quarantine.

Main outcome measures Positive for SARS-CoV-2 virus on reverse transcription PCR with self-administered oralnasal swab and general well-being using the WHO-5 Wellbeing Index.

Results Of 16361 passengers enrolled, 248 (1.5\%, $95 \% \mathrm{Cl} 1.3 \%$ to $1.7 \%)$ tested positive. Of these, $167(67 \%)$ were identified on arrival, 67 (27\%) on day 7, and 14 $(6 \%)$ on day 14 . The positivity rate increased from $1 \%$ in September to $2 \%$ in October. Average well-being score declined from 19.8 (out of a maximum of 25) to 15.5 between arrival and day $7(p<0.001)$.

Conclusions A single arrival test will pick up two-thirds of individuals who will become positive by day 14 , with most of the rest detected on the second test on day 7 . These results support strategies identified through mathematical models that a reduced quarantine combined with testing can be as effective as a 14-day quarantine.

\section{INTRODUCTION}

As COVID-19 has rapidly spread across the globe and threatened the lives and safety of people in all regions, governments have attempted to find means to limit transmission, often relying on limited knowledge and evidence. Measures to control disease spread across international borders have

\section{Strengths and limitations of this study}

- Decisions regarding border restrictions have been based on trial and error and mathematical models with limited empirical data to support such decision-making.

- This study assessed the prevalence of SARS-CoV-2 in a cohort of international travellers on arrival and on day 7 and day 14 of quarantine.

- It is limited to one airport and there is potential from bias due to non-participation and loss to follow-up.

- Self-collected oral-nasal swabs were used, which facilitated participation but may have reduced sensitivity.

included identification of ill passengers by symptom screening or temperature checks, strict quarantine requirements, or combinations of virus testing and quarantine. Many countries, such as Canada, have kept borders closed to foreign travellers, with the exception of essential workers and returning citizens and permanent residents. ${ }^{1}$ This, coupled with a 14-day quarantine requirement, was designed to discourage international travel and reduce the risk of imported COVID-19 from abroad. Other countries have adopted strategies that either require a predeparture test, arrival and/or postarrival test combined with reduced or no quarantine. Some countries have also taken a risk-based approach, with varying requirements dependent on the risk of COVID-19 transmission in the origin country or the activities the traveller will engage in. Decisions regarding such approaches have largely been based on trial and error or mathematical modelling. ${ }^{2-7} \mathrm{~A}$ recent Cochrane review concluded that the quality of evidence for most travel control measures was very low with mixed results, and that the optimal approach likely depends on a specific country's context and own epidemiology. ${ }^{8}$ 
Table 1 Baseline characteristics by case counts

\begin{tabular}{|c|c|c|c|c|c|}
\hline & & & Case & & \\
\hline & Responses, $n=16361$ & Imputed, $n=16361$ & Yes, $n=248(1.5 \%)$ & No, $n=16113(98 \%)$ & P value* \\
\hline Gender, n (\%) & & & & & 0.13 \\
\hline Male & $5159(31.5)$ & $8289(51)$ & $141(57)$ & $8148(51)$ & \\
\hline Other & $17(<0.1)$ & $17(0.1)$ & $0(0)$ & $17(0.1)$ & \\
\hline Age category, n (\%) & & & & & 0.55 \\
\hline $18-29$ & 3014 (18.4) & $5012(31)$ & $88(35)$ & $4924(31)$ & \\
\hline $30-49$ & $4266(26.1)$ & $6915(42)$ & $100(40)$ & $6815(42)$ & \\
\hline $50-69$ & 2498 (15.3) & $4121(25)$ & $56(23)$ & $4065(25)$ & \\
\hline $70-79$ & $175(1.1)$ & $298(1.8)$ & $4(1.6)$ & $294(1.8)$ & \\
\hline Africa & $524(3.2)$ & $661(4.0)$ & $15(6.0)$ & $646(4.0)$ & \\
\hline America & $6629(40.5)$ & $9165(56)$ & $120(48)$ & $9045(56)$ & \\
\hline Asia & $1738(10.6)$ & $2176(13)$ & $53(21)$ & $2123(13)$ & \\
\hline Europe & 3584 (21.9) & $4315(26)$ & $60(24)$ & $4255(26)$ & \\
\hline Oceania & $44(0.3)$ & $44(0.3)$ & $0(0)$ & $44(0.3)$ & \\
\hline Unknown & $3842(23.5)$ & - & - & - & \\
\hline Risk category, n (\%) & & & & & 0.21 \\
\hline Green & $692(4.2)$ & $796(4.9)$ & $8(3.2)$ & $788(4.9)$ & \\
\hline
\end{tabular}

*Statistical tests performed: Fisher's exact test; $\chi^{2}$ test of independence.

Quarantines can be difficult to enforce, have variable compliance and may result in significant negative effects related to social isolation, restricted physical activity, lost productivity and income. ${ }^{9-11}$ Direct impacts of border closures and travel hesitancy related to quarantine are felt in the travel industry, which represents a large portion of the global economy. ${ }^{12} 13$

While testing of infected travellers should reduce the risk of disease importation, SARS-CoV-2 presents challenges given the potential for asymptomatic and presymptomatic transmission. Symptom screening and temperature checks will not detect asymptomatic or presymptomatic individuals. Testing, either predeparture or on arrival, will miss those individuals who have been just infected and are still incubating the virus. Thus, quarantine remains one of the few options, but the optimal length of quarantine remains unclear. One modelling study suggests that quarantine with testing on day 7 achieves a level of risk reduction similar to that of a 14-day quarantine. ${ }^{14}$ This assumes that there is perfect compliance and/or enforcement of the quarantine measures.
Given the substantial costs and the impact of quarantine on personal well-being, it is critical to generate empirical data to support theoretical and mathematical models. To our knowledge, there are no systematic data reported on the proportion of international travellers that test positive for SARS-CoV-2 on arrival to Canada and during quarantine. The present study aimed to systematically estimate the positivity rate of air travellers coming to Toronto, Canada on arrival and on day 7 and day 14 in September and October 2020. A further objective was to determine whether a combination of testing and country of origin could accurately identify those who were at highest risk of developing COVID-19. We also assessed knowledge and attitudes towards COVID-19 control measures and subjective well-being during the quarantine period.

\section{METHODS}

Study design

We conducted a prospective cohort study of arriving international travellers to Toronto Pearson International 
17,744

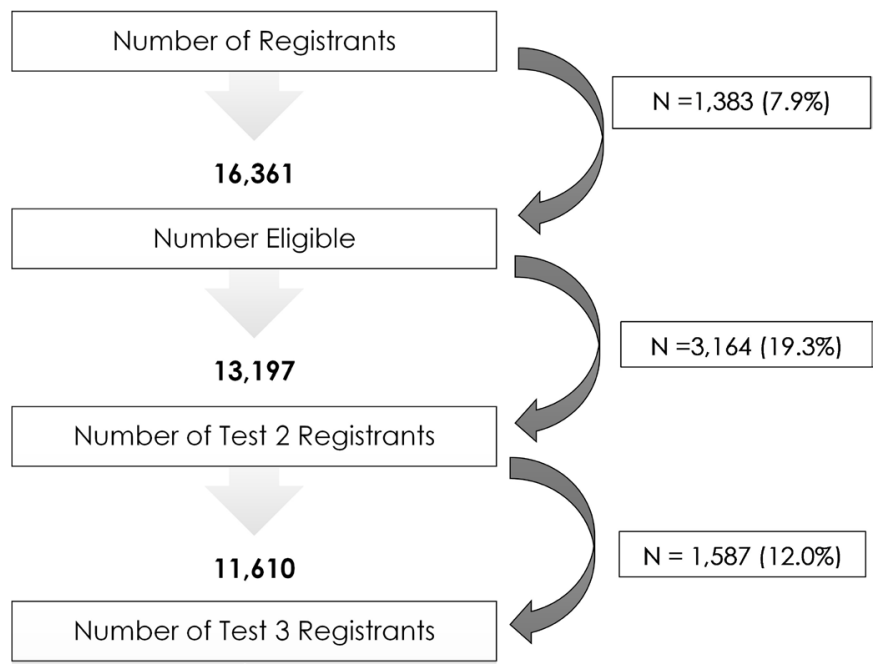

Figure 1 Participation flow chart.

Airport, Terminal 1 between 3 September 2020 and 31 October 2020.

Inclusion criteria were those aged 18 or older who had a final destination within $100 \mathrm{~km}$ of Toronto Pearson Airport, could speak English or French, and provided consent. Exclusion criteria were passengers taking a connecting flight through Pearson Airport, had no internet access, exhibited symptoms of COVID-19 on arrival or were exempted from quarantine (eg, essential workers).

\section{Patient and public involvement}

The study does not involve patients. As the study was mounted rapidly there was limited opportunity for broad public engagement prior to the study launch. The partner airlines and airport had engagement with current and prospective travellers whose perspectives were used to plan the study. A pilot phase assessed traveller interest in and concerns about the study and its materials. The interim and final results have been publicly disseminated and made available to the participants.

\section{Enrolment procedures}

Individuals arriving on flights with participating Star Alliance airlines were invited to join the study during their flight or after arrival. Flights from any international destination that terminated at Pearson International Airport Terminal 1, Toronto, Ontario, were included. Flight crew announced the opportunity to participate in the study and directed passengers to view an instructional video that was prepared by the investigators in English and French on the inflight entertainment systems. Flight crews were given a script to answer basic questions from potential participants, such as how to get to the study booth after landing, but referred interested individuals to the investigator team's research personnel for any questions related to the study. Information and study invitations were also posted in the arrivals and baggage areas of Terminal 1 in order to ensure that interested passengers could directly review and consider study materials after arrival.

On arrival, eligible and consenting passengers were guided to the testing booths. These were located in a secure area, after passage through immigration and customs, and following baggage collection. After reviewing a study information sheet and completing informed consent, participants proceeded to a specimen collection booth, where they were trained and supervised in the self-collection of oral-nasal swabs. Briefly, a flocked swab (Miraclean, Shenzhen, China) was moistened on the tongue, followed by bilateral swabbing of the buccal sulcus (between the cheeks and the gums) with rotation three times, followed by insertion into each nostril approximately $2-4 \mathrm{~cm}$ as a 'deep nasal' swab and rotated three times. Swabs were placed in $2 \mathrm{~mL}$ McMaster Molecular Medium (MMM), a guanidine isothiocyanate-based lysis buffer designed to inactivate the virus and preserve RNA (Research St Joseph's, Hamilton Ontario). Participants were given two further specimen sampling kits for selfadministration on days 7 and 14. For the two remaining tests after arrival, couriers were arranged to pick up the kits at the passengers' location of quarantine.

The participants completed online questionnaires at the same three timepoints. Prompts were provided by SMS (short message service)/text message to complete the follow-up tests and questionnaires. The questionnaires covered travel history, symptoms, mental health, attitudes towards protection measures (eg, quarantine) and behaviours (eg, handwashing). The items were drawn from the WHO survey tool and guidance for rapid, simple, flexible behavioural insights on COVID-19. ${ }^{15}$

\section{Laboratory methods}

Swabs collected in MMM were batch-processed from nucleic acid extraction to reverse transcription quantitative real-time PCR (RT-qPCR) set-up on the Hamilton Microlab STAR (Hamilton Company, Nevada, USA). Nucleic acid extraction was performed using the Maxwell HT Viral TNA Kit (Promega, Wisconsin, USA). For RT-qPCR, Luna Universal Probe One-Step RT-qPCR (New England BioLabs, Massachusetts, USA) was used in combination with custom synthesised primers and probes (LGC Biosearch, California, USA). RT-qPCR was performed for 45 cycles on all specimens on the Bio-Rad CFX96 Touch Real-Time PCR Detection System (BioRad Laboratories, California, USA) using the laboratorydeveloped triplex assay, which contains two SARS-CoV-2 targets (envelope, 5'-untranslated region (5'-UTR)) and a human housekeeping/sample adequacy target, RNase P. All PCR testing were conducted at the Research Institute of St Joseph's in Hamilton, Ontario. As testing was done in a research laboratory, in order to ensure appropriate confirmation and reporting to public health those who were determined 'non-negative' were then referred to a provincial government COVID-19 assessment centre for a nasopharyngeal swab. For clarity, those who tested 
Table 2 Demographics of passengers lost to follow-up

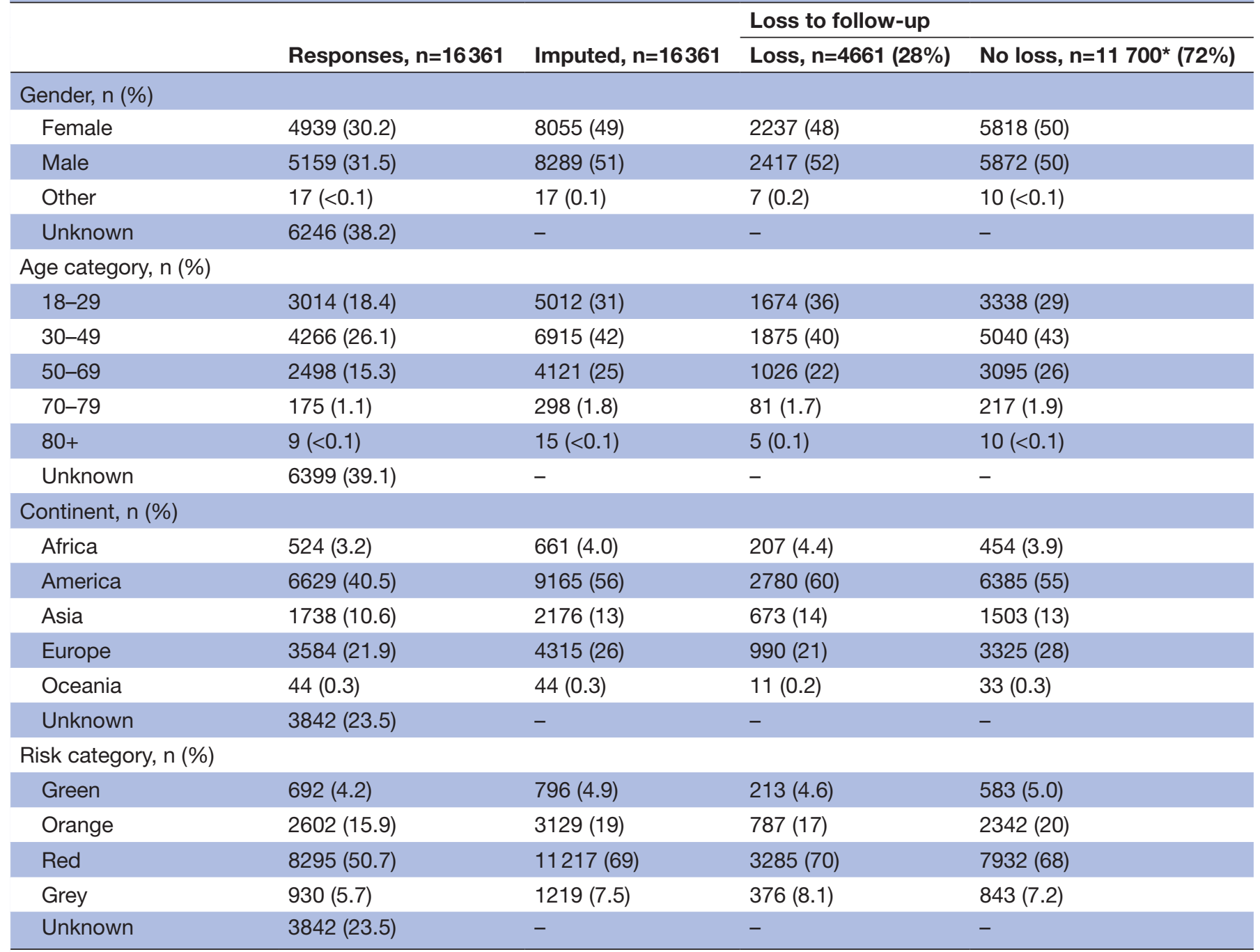

*Passengers who tested positive for COVID-19 were counted as completing the study irrespective of how many follow-up tests they completed considering disease status is known.

'non-negative' in the research laboratory will be referred to as 'positives' throughout the manuscript.

Viral load, as measured by the average cycle threshold (Ct) during PCR for envelope gene and 5'-UTR, was divided a priori into high viral load (Ct $<25$ cycles), moderate viral load (Ct 25-35 cycles) or low viral load ( $>35$ cycles). All viral loads were obtained from duplicate PCR measurements, and where an analyte was not amplified a value of 45 was imputed.
Data management and analysis

All participant information and laboratory results were stored in a secure cloud-based information system.

The main endpoints were rate of travellers testing positive for the first time on arrival and on day 7 and day 14 . Exact $95 \%$ CIs for the proportion of travellers testing positive were calculated using the Clopper-Pearson exact method. The denominator for calculating proportion positive by timepoint was the total number of participants

Table 3 Primary results

\begin{tabular}{llllll}
\hline Time & Cases & $\mathbf{n}$ & Rate per $\mathbf{1 0 0 0 0 0}$ & Lower $\mathbf{C l}$ & Upper $\mathbf{C l}$ \\
\hline Overall & 248 & 16361 & 1516 & 1334 & 1715 \\
Arrival & 167 & 16361 & 1021 & 872 & 1187 \\
Day 7 & 67 & 13197 & 508 & 394 & 644 \\
Day 14 & 14 & 11610 & 121 & 66 & 202 \\
\hline
\end{tabular}




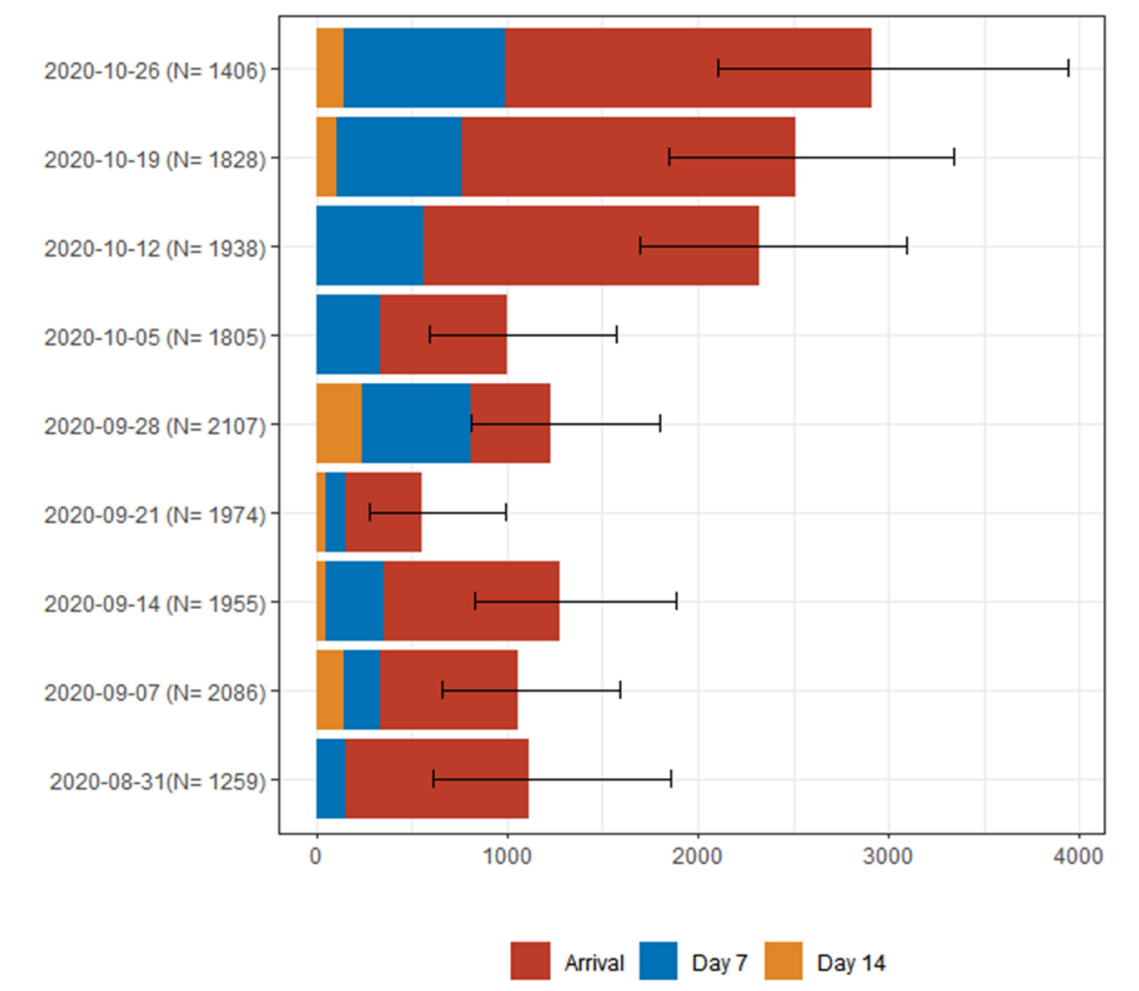

Note: Test numbers indicate rates for when passengers first became positive for COVID-19.

Figure 2 Rates of COVID-19 infections by week of arrival.

who registered a test for the respective timepoint (ie, arrival, day 7 and day 14). In order to account for individuals who did not complete follow-up tests, two approaches were taken. First, a sensitivity analysis was conducted by assuming different positivity rates based on best-case and worst-case scenarios for those lost to follow-up. We conducted a second analysis where we used inverse probability weighting (IPW) to adjust for potential selection bias associated with dropouts. Specifically, we developed two IPW models using logistic regression to assess differences between those who dropped out on day 7 and day 14 versus those who did not and adjusted for positivity rates using the weights. ${ }^{16}$ Regarding our IPW approach, as weighted estimates no longer follow a binomial distribution, bootstrap $95 \%$ CIs were used instead. ${ }^{17}$

Baseline descriptive statistics and measures of independence were computed for key demographic and travelrelated variables. Country of origin was classified based on the European Union risk framework. ${ }^{18}$ This classifies countries as low, medium and high risk based on incidence and test positivity rates. Countries with insufficient information or a testing rate of less than 300 per 100 000 population per week are classified as grey. Daily data posted by the European Centre for Disease Prevention and Control were used to assess each country's risk on the participant's arrival date.

General well-being was captured with the 5-item WHO Well-being Index, which is a validated tool to measure mental health and well-being. ${ }^{15}$ The items address feelings of cheerfulness, calm, interest in one's life, vigour and well rested over the previous 2 weeks. Each item is scored from 0 (never) to 5 (all the time), with a maximum theoretical score of 25.

As some participants did not complete all items on the questionnaires, we used a multiple imputation (MI) approach to impute missing values. MI uses logistic and multinomial logistic regression to create multiple data sets of predicted values and takes the average across data sets as the final imputed value. Variables used in this approach were gender, age, continent of origin, mental health, risk category and handwashing (a behaviour variable). In case of missing country of origin data, a grouped imputation approach was used. Given the large variance of responses, MI was not possible. Therefore, groups of 20 travellers that arrived at the study booth at the same time were made around missing values and the most frequent country of origin for these groups was imputed. This approach assumes that registrants usually arrive in groups as they are recruited on their respective flights.

Given the low prevalence expected on the final test, essentially zero, we used the method outlined in Frank et $a l^{19}$ for assessing our power. A sample size of 10000 completed day 14 tests was selected to be sufficient to rule out the true proportion being $100 \%$ greater than that observed. 
Table 4 Symptomatic status of positives by timepoint

\begin{tabular}{|c|c|c|c|}
\hline & \multicolumn{3}{|l|}{ Test } \\
\hline & Arrival, $\mathrm{n}=167(67 \%)^{*}$ & Day $7, \mathrm{n}=67(27 \%)^{*}$ & Day $14, n=14(5.6 \%)^{*}$ \\
\hline \multicolumn{4}{|c|}{ Arrival symptoms } \\
\hline No & $72(96)$ & $31(100)$ & $6(100)$ \\
\hline Unknown & 92 & 36 & 8 \\
\hline Yes & $30(40)$ & $12(30)$ & $0(0)$ \\
\hline No & $45(60)$ & $28(70)$ & $11(100)$ \\
\hline Unknown & 92 & 27 & 3 \\
\hline \multicolumn{4}{|c|}{ Day 14 symptoms } \\
\hline Yes & $14(23)$ & $14(44)$ & $3(38)$ \\
\hline
\end{tabular}

*Statistics presented: $\mathrm{n}(\%)$.

\section{RESULTS}

\section{Study population}

A total of 16361 passengers registered for the study and completed at least one test. Study participants arrived from all continents (except Antarctica) and represented all risk categories and age groups. The highest proportion of participants were those arriving from the Americas $(56 \%)$, countries classified as 'red' according to the European Union risk classifications (69\%), and younger and middle age groups ( $73 \%$ between the ages of 18 and 49) (table 1).

Approximately $8 \%-20 \%$ of the cohort were lost to follow-up at the various timepoints (figure 1). Participants lost to follow-up were more likely to be in the youngest age group ( $36 \%$ between the ages of 18 and $29)$, arriving from the Americas $(60 \%)$ or arriving from a red risk country (70\%) (table 2). The USA was overrepresented as the country of origin among participants lost to follow-up.

\section{Symptoms and positive COVID-19 results}

Throughout the study period, 248 (1.5\% of those completing the first test, $95 \%$ CI $1.3 \%$ to $1.7 \%$ ) individuals who tested positive at least once were identified. Of these cases, $167(67 \%)$ were identified on arrival, $67(27 \%)$ on day 7 , and $14(6 \%)$ on day 14 of quarantine (table 3 ). The proportion of positive participants increased from early September to end of October (figure 2).

Of the 167 individuals who tested COVID-19-positive on arrival, 3 participants reported symptoms on arrival, 30 participants on day 7 and 14 participants on day 14 (table 4). At each time period, a higher proportion of those who tested positive were asymptomatic or presymptomatic. Note that the three participants who reported symptoms on the arrival questionnaire were not excluded from the initial screening due to the possible time lag between entering the study and answering the online questionnaire. Therefore, these participants could have been asymptomatic at the time the test was administered and then developed symptoms later that day when they filled out the online questionnaire. All participants had to have reported no symptoms to the border officer in order to be eligible for the study, since anyone reporting symptoms on arrival was immediately referred to a quarantine officer.

Passengers who tested positive during the study period were more likely to be in the younger age groups, male $(57 \%)$ and to be arriving from countries classified as 'red' in terms of risk (figure 3). In comparison with those who tested negative during the study period, the highest proportion of positive travellers came from 'grey' risk countries $(7.4 \%$ vs $8.9 \%)$.

In order to address the possibility of missed cases from the travellers who were lost to follow-up, $1 \%$ of these losses were added to the overall case counts for day 7 and day 14 . After this analysis was run, the conservative case count estimate for the entire study period was 292, with 107 of these cases being picked up on day 7 and 18 on day 14 (table 5). Similar results were obtained with the IPW method.

\section{Viral load estimation}

The level of viral load in positive cases is presented in table 6. Low viral load was present on arrival in 60 out of $167(36 \%)$ positive individuals, 20 out of $67(30 \%)$ on day 7 , and 6 out of 14 (43\%) on day 14 . Only 2 of the 14 positives on day 14 had a high viral load.

\section{Well-being and attitudes regarding COVID-19 and public health approaches}

The average mental health score was 19.8 on arrival and dropped to 15.5 and 15.2 on days 7 and 14, respectively. Participants had a much more favourable disposition on arrival than during quarantine. 


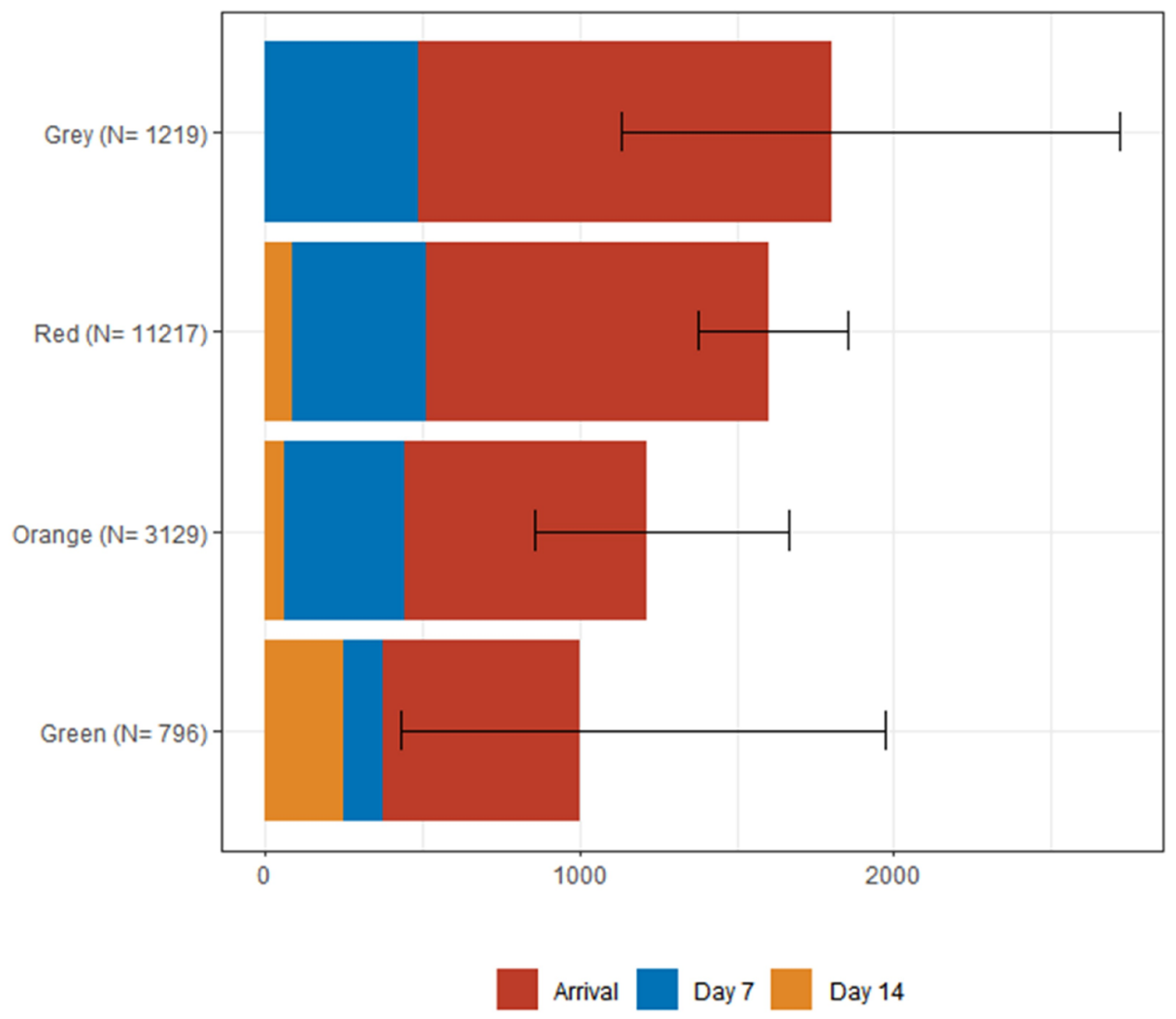

Note: Test numbers indicate rates for when passengers first became positive for COVID-19.

Figure 3 Rates of COVID-19 infections by European Centre for Disease Prevention and Control risk category.

Table 7 presents two keyattitudinal items regarding controls for international travel. Participants were more willing to accept a requirement for testing than for immunisation.

\section{DISCUSSION}

In order to control the spread of COVID-19 globally, governments across the world are resorting to the use of travel restrictions and quarantine. In today's globalised

Table 5 Sensitivity analysis to address loss to follow-up

\begin{tabular}{|c|c|c|c|c|}
\hline Time & Cases & $\begin{array}{l}\text { Rate per } \\
100000\end{array}$ & Lower $\mathrm{Cl}$ & Upper Cl \\
\hline \multicolumn{5}{|c|}{ Adding $1 \%$ of those lost to follow-up } \\
\hline Overall & 292 & 1785 & 1587 & 1999 \\
\hline Arrival & 167 & 1021 & 872 & 1187 \\
\hline Day 7 & 107 & 654 & 536 & 790 \\
\hline Day 14 & 18 & 110 & 65 & 174 \\
\hline
\end{tabular}

Inverse probability weighted estimation

\begin{tabular}{lllll} 
Overall & 272 & 1662 & 1455 & 1858 \\
Arrival & 167 & 1021 & 862 & 1179 \\
Day 7 & 84 & 519 & 404 & 627 \\
Day 14 & 21 & 130 & 73 & 185 \\
\hline
\end{tabular}

environment in which travel is ubiquitous and central to many national economies, it is critical to determine which measures are most appropriate. This study aimed to systematically estimate the positivity rate, symptoms, attitudes and well-being of travellers on arrival in Toronto, Canada and during their 14-day quarantine in the fall of 2020.

Results revealed that $1.5 \%$ of the study participants arriving as international passengers at Toronto Pearson Airport tested positive by reverse transcription PCR on or after arrival. The overall rates were approximately $1 \%$ in September and 2\% in October, reflecting the rapidly changing conditions in the USA and Europe, which were the regions of origin for the largest proportion of arriving passengers. Approximately two-thirds of positive cases were detected on arrival, with most of the remaining cases being identified on day 7 . Of the small number that were positive on day 14 , approximately half had very low viral loads, suggesting that their positive status may have been an artefact of previous infection and did not reflect active infection.

Men had higher positivity rates, although not statistically significant, than women.

These results support those from modelling studies and the December 2020 recommendations from the US Centers for Disease Control and Prevention that a 
Table 6 Cycle threshold (Ct) as surrogate for RNA load by first positive test

\begin{tabular}{|c|c|c|c|}
\hline & \multicolumn{3}{|l|}{ Test } \\
\hline & Arrival, $n=167^{\star}$ & Day $7, n=67^{*}$ & Day $14, n=14^{*}$ \\
\hline Average $\mathrm{Ct}^{\star}$ across all targets & $32(26-38)$ & $27(22-36)$ & $30(27-37)$ \\
\hline \multicolumn{4}{|l|}{ Viral load } \\
\hline Low (Ct >35) & $60(36)$ & $20(30)$ & $6(43)$ \\
\hline Moderate (Ct 25-35) & $71(43)$ & $21(31)$ & $6(43)$ \\
\hline High $(\mathrm{Ct}<25)$ & $36(22)$ & $26(39)$ & $2(14)$ \\
\hline
\end{tabular}

*Statistics presented: median (IQR) or $\mathrm{n}(\%)$.

shortened quarantine period of 7 days combined with a negative test would provide the same degree of control as a 14-day quarantine. ${ }^{20}$ This is particularly important given the significant impact observed on general well-being among the participants-consistent with other studies. ${ }^{9}$ However, such a recommendation assumes perfect compliance with quarantine, which is difficult to achieve unless very strict requirements, such as specialised quarantine facilities or global positioning system tracking, are implemented. ${ }^{11}$ It is plausible that compliance with a 7-day quarantine and test regimen may be better than a 14-day quarantine.

A relationship with risk of COVID-19 in the origin country was observed. However, the differences between risk categories were small and the benefits of a risk-based approach may be marginal. Furthermore, countries move rapidly through the different risk categories, which makes implementation of such approaches difficult.
These results support policy development regarding testing and quarantine duration. Policy regarding implementation of restrictions for specific countries or regions will need to continue to rely on models. For example, Russell et $a l^{21}$ have modelled when travel restrictions might have the greatest impact. They show that stringent restrictions will be most effective for countries with low COVID-19 incidence and large numbers of arrivals from other countries, or where epidemics are close to tipping points. They recommend that countries should consider local COVID-19 incidence, local epidemic growth and travel in determining appropriate restrictions.

In assessing whether or not to use testing to replace or reduce quarantine, another important consideration is the availability of testing. In settings where availability of testing is limited, prioritising border testing in order to reduce quarantine time may not be warranted. However, with the emergence of variants of concern, surveillance

Table 7 Baseline attitude responses

\begin{tabular}{|c|c|c|}
\hline & Case & \\
\hline & Yes, $n=248^{*}$ & No, $n=16113^{*}$ \\
\hline COVID-19 test required $\dagger$ & & \\
\hline Very acceptable & $54(50)$ & $5513(57)$ \\
\hline Acceptable & $40(37)$ & $3021(31)$ \\
\hline Neither acceptable nor unacceptable & $7(6.5)$ & $804(8.2)$ \\
\hline Unacceptable & $5(4.7)$ & 285 (2.9) \\
\hline Very unacceptable & $1(0.9)$ & $132(1.4)$ \\
\hline Unknown & 141 & 6358 \\
\hline Vaccination required $\neq$ & & \\
\hline Very acceptable & $33(31)$ & 3685 (38) \\
\hline Acceptable & $33(31)$ & $2631(27)$ \\
\hline Neither acceptable nor unacceptable & $25(24)$ & $1615(17)$ \\
\hline Unacceptable & $7(6.6)$ & $928(9.6)$ \\
\hline Very unacceptable & $8(7.5)$ & $828(8.5)$ \\
\hline Unknown & 142 & 6426 \\
\hline
\end{tabular}

*Statistics presented: $\mathrm{n}(\%)$.

†'If a negative COVID-19 test were required for international travel in the future how acceptable would you find that?'

‡'If proof of a COVID-19 vaccination were required for international travel in the future, how acceptable would you find that?' 
testing at borders may need to be increased. Indeed, many countries have tightened border controls, including quarantine and testing requirements, following the identification of variants of concern.

\section{Limitations}

This study was conducted at a single terminal at Toronto's Pearson Airport, although representing the majority of international flights arriving at Canada's busiest airport. We enrolled approximately $20 \%$ of passengers. We believe that up to half of the arriving passengers would have met our exclusion criteria, so our participation rate likely approached $40 \%$. There will likely have been selection bias in our participants. However, it is uncertain what the direction may have been. It may be possible that those who engaged in higher risk behaviours while abroad may have chosen not to participate. On the other hand, during the study period, PCR testing was not broadly available in Ontario, and some participants told us they took part in order to access the free testing. Regardless, selection bias would affect the overall positivity rate. However, a key value of our results is the distribution of positivity across the three timepoints, which should not be affected by selection bias. We do not know if any of our participants became positive after day 14, although it is unlikely that those with mild to moderate COVID-19, which is what would be expected in our cohort, present so late. ${ }^{22}$

We had losses to follow-up, and these may have biased the results if those who broke quarantine or developed symptoms might have been less likely to participate or less likely to follow up. We adjusted using a sensitivity analysis and IPW, with similar results using both methods. IPW were based only on the variables that were measured and thus selection bias due to unmeasured factors may not be accounted for. The overall conclusions are not changed with either method.

We used supervised self-collection at the airport and unsupervised self-collection at home for follow-up. Measurement of RNase P levels found inadequate levels in only $0.2 \%$, suggesting the approach enabled adequate sample collection in the vast majority of participants. The correlation between oral-nasal self-collection and staffadministered nasopharyngeal collection is estimated at $90 \%-95 \%$ sensitivity, and the use of serial weekly collection on three occasions over 14 days of quarantine may have increased sensitivity.

\section{CONCLUSIONS}

We demonstrated the feasibility of large-scale selfcollection of oral-nasal swabs, coupled with a highly sensitive, laboratory-based PCR testing, for arrival and follow-up testing of international passengers. Approximately $94 \%$ of infections were identified through arrival and day 7 testing, confirming findings from mathematical models that a 7-day quarantine coupled with testing would be highly effective in identifying importation of COVID19. Given concern about importation of spike-protein variants of potential public health significance, airport testing would enable more timely detection and tracing of imported COVID-19 variants.

\section{Twitter Laura C Rosella @LauraCRosella}

Contributors All authors contributed to the development and implementation of the study, review of results, and preparation and approval of the manuscript. MJ supervised the recruitment of participants and acquisition of data. MS and DB supervised the laboratory analysis. MS and DM contributed infectious disease expertise to the study design and results interpretation. VG and LCR contributed public health and epidemiology expertise to the study design and results interpretation. CR supervised the collection and interpretation of psychosocial data. VG, LCR and EDP conducted the data analysis and verified the underlying data.

Funding The conduct of the study was protected from the role of the funders. McMaster HealthLabs (MHL) is a not-for-profit corporation created by McMaster University and Research St Joseph's to conduct COVID-19-related laboratory research. The contracts for funding with Air Canada and the Greater Toronto Airports Authority were with MHL. The funders also provided inkind support for recruitment of participants and the study site at the airport. The Canadian Institutes of Health Research awarded a peer-reviewed grant for conduct of the study. The funders did not have any direction in the study design, execution, data analysis or preparation of the manuscript.

Competing interests MS and DB are on the Board of Directors of McMaster HealthLabs. DB is the developer of McMaster Molecular Medium.

Patient and public involvement Patients and/or the public were not involved in the design, or conduct, or reporting, or dissemination plans of this research.

Patient consent for publication Not required.

Ethics approval The study protocol was reviewed and approved by the Advarra Research Ethics Board.

Provenance and peer review Not commissioned; externally peer reviewed.

Data availability statement Deidentified participant data are available through an open data repository available at https://doi.org/10.5683/SP2/L0Q2HQ.

Open access This is an open access article distributed in accordance with the Creative Commons Attribution Non Commercial (CC BY-NC 4.0) license, which permits others to distribute, remix, adapt, build upon this work non-commercially, and license their derivative works on different terms, provided the original work is properly cited, appropriate credit is given, any changes made indicated, and the use is non-commercial. See: http://creativecommons.org/licenses/by-nc/4.0/.

\section{ORCID iDs}

Vivek Goel http://orcid.org/0000-0002-1389-2007

Laura C Rosella http://orcid.org/0000-0003-4867-869X

Dominik Mertz http://orcid.org/0000-0003-4337-1613

Cheryl Regehr http://orcid.org/0000-0001-7814-7836

\section{REFERENCES}

1 Government of Canada. Coronavirus disease (COVID-19): travel restrictions, exemptions and advice, 2020. Available: https:// www.canada.ca/en/public-health/services/diseases/2019-novelcoronavirus-infection/latest-travel-health-advice.html\#_Mandatory_ quarantine_and

2 Nicholson P, Goel V, Larsen J. COVID-19 and Canada's border. Public Policy Forum, 2021. Available: https://ppforum.ca/ publications/covid-19-and-canadas-border/

3 Quilty BJ, Clifford S, Flasche S, et al. Effectiveness of Airport screening at detecting travellers infected with novel coronavirus (2019-nCoV). Euro Surveill 2020;25:2000080.

4 Wong J, Abdul Aziz ABZ, Chaw L, et al. High proportion of asymptomatic and presymptomatic COVID-19 infections in air passengers to Brunei. J Travel Med 2020;27:taaa066.

5 Wang CJ, Ng CY, Brook RH. Response to COVID-19 in Taiwan: big data analytics, new technology, and proactive testing. JAMA 2020;323:1341.

6 Schengen Visalnfo News. Eu countries increase COVID-19 travel measures. SchengenVisalnfo.com, 2020. Available: https://www. schengenvisainfo.com/news/eu-countries-increase-covid-19-travelmeasures/ 
7 Government of Iceland. New rules on quarantine, isolation and screening at the border, 2021. Available: https://www.government. is/news/article/2020/08/18/New-rules-on-quarantine-isolation-andscreening-at-the-border/

8 Burns J, Movsisyan A, Stratil JM, et al. Travel-related control measures to contain the COVID-19 pandemic: a rapid review. Cochrane Database Syst Rev 2020:Issue 9. Art. No.: CD013717.

9 Brooks SK, Webster RK, Smith LE, et al. The psychological impact of quarantine and how to reduce it: rapid review of the evidence. The Lancet 2020;395:912-20.

10 Pfefferbaum B, North CS. Mental health and the Covid-19 pandemic. N Engl J Med 2020;383:510-2.

11 Webster RK, Brooks SK, Smith LE, et al. How to improve adherence with quarantine: rapid review of the evidence. Public Health 2020;182:163-9.

12 IATA. Traveler survey reveals COVID-19 concerns, 2020. Available: https://www.iata.org/en/pressroom/pr/2020-07-07-01/

13 World Tourism Organization. International tourist numbers could fall $60-80 \%$ in 2020, 2020. Available: https://www.unwto.org/news/ covid-19-international-tourist-numbers-could-fall-60-80-in-2020

14 Clifford S, Quilty BJ, Russell TW, et al. Strategies to reduce the risk of SARS-CoV-2 re-introduction from international travellers. medRxiv 2020:2020.07.24.20161281.

15 World Health Organization, Regional office for Europe. Survey tool and guidance: rapid, simple, flexible behavioural insights on
COVID-19, 2020. Available: https://apps.who.int/iris/handle/10665/ 333549

16 Howe CJ, Cole SR, Lau B, et al. Selection bias due to loss to follow up in cohort studies. Epidemiology 2016;27:91-7.

17 Davison AC, Hinkley DV. Bootstrap methods and their application. Cambridge: Cambridge University Press, 1997.

18 European Commission. A common approach to travel measures in the EU. Centre for disease prevention and control and European Union aviation safety agency, 2021. Available: https://ec.europa. eu/info/live-work-travel-eu/coronavirus-response/travel-duringcoronavirus-pandemic/common-approach-travel-measures-eu_en

19 Frank JW, Coates RA, Brant R, et al. Sample sizes for needles in a haystack: the case of HIV seroprevalence surveys. Can J Public Health 1990;81:50-2.

20 Centers for Disease Control and Prevention. Options to reduce quarantine for contacts of persons with SARS-CoV-2, 2020. Available: https://www.cdc.gov/coronavirus/2019-ncov/more/ scientific-brief-options-to-reduce-quarantine.html

21 Russell TW, Wu JT, Clifford S, et al. Effect of internationally imported cases on internal spread of COVID-19: a mathematical modelling study. Lancet Public Health 2021;6:e12-20.

22 Center for Disease Control and Prevention. Interim guidance on ending isolation and precautions for adults with COVID-19, 2021. Available: https://www.cdc.gov/coronavirus/2019-ncov/hcp/durationisolation.html 